\title{
A Study of the Structure Formed by Thickeners of Greases Using Atomic Force Microscope
}

\author{
Michita Hokao $^{1)^{*}}$, Noriyuki Inami ${ }^{1)}$, Eri Watabe ${ }^{1)}$, Atsushi Yokouchi ${ }^{1)}$ and Joichi Sugimura ${ }^{2)}$ \\ ${ }^{1)}$ Corporate Research \& Development Center, NSK Ltd. \\ 1-5-50 Kugenumashinmei, Fujisawa, Kanagawa 251-8501, Japan \\ ${ }^{2)}$ Department of Mechanical Engineering, Kyushu University \\ 744 Motooka, Nishi-ku, Fukuoka 819-0395, Japan \\ *Corresponding author: hokao@nsk.com

\begin{abstract}
( Manuscript received 8 May 2012; accepted 11 November 2012; published 31 January 2013 )
(Presented at Symposium S4: The Advanced Lubricating Grease Technology in the International Tribology Conference Hiroshima 2011)
\end{abstract}

\begin{abstract}
This paper describes an experimental study on quantitative evaluation of correlation between the structure formed by thickeners and yield stress of greases. In order to evaluate the thickener structures, untreated surface of greases was observed by using an atomic force microscope, and an index was obtained from AFM images to describe the degree of dispersion of the thickeners. The index physically represented both factors of shape and distribution of thickeners. The results for twelve types of lithium soap greases with different base oils and thickener concentrations showed good correlation between the index of the degree of dispersion and the yield stress of the greases. This relationship indicated that uniform distribution of thickeners in greases caused higher yield stress.
\end{abstract}

Keywords: grease, structure, atomic force microscope, yield stress, thickeners, lithium soap, degree of dispersion

\section{Introduction}

The structure of thickeners formed in greases is one of the key factors in grease lubrication. The three-dimensional structure formed by thickeners holds and provides base oil to lubricated contact, and also affects flow capability of grease. In addition, it works to provide boundary lubricating film of the thickeners themselves. It is therefore important to understand the structure of the thickeners and their behaviors for proper design of grease lubrication.

The authors have recently studied effects of thickeners on mechanical properties and tribological performance of greases. They have shown that lithium soap grease with longer soap fibers has higher entrainment capability into the conjunction and exhibits lower friction coefficient in boundary lubrication [1]. They have also shown that lithium soap grease with denser structure has higher yield stress and provides lower steady-state bearing torque [2].

Thickener fibers distributed in the grease form three-dimensional structure, and oil is held by a combination of interactive force and capillary force within the pockets formed by the network [3]. This structure is deformed depending on amplitude of shear stress. As is well known, greases are viscoelastic materials which have yield stress and show shear thinning [4]. The complicated rheological properties of greases are supposed to be originated from the thickener structure characteristics.

The influence of three-dimensional structure on rheological characteristics has been investigated qualitatively by some groups. Couronné et al. observed microstructures of lithium soap, lithium complex soap and urea greases using transmission electron microscope (TEM) and discussed influences of microstructure on the rheological behavior [5]. Delgado et al. pointed out influences of grease microstructure observed by scanning electron microscope (SEM) on the rheological characteristics of the lithium soap greases with different thickener concentrations and base oil viscosities [6]. In these SEM and TEM studies, they made pre-treatment to remove base oil from greases. Consequently, they observed only shape and configuration of fibers at dried condition, and discussed the three dimensional structure qualitatively. In order to explore the thickener structures with interactive forces between thickener and base oils present, it may be necessary to make a direct observation 
of untreated greases. It is also necessary to describe observation results quantitatively so as to be used to understand the relationship between the thickener structures and behaviors of greases.

An observation in ambient air is required for detailed investigation of the thickener structures. Atomic force microscope (AFM) is one of the most popular methods to observe solid surface with high resolution in ambient air, and is suitable for the observation of the structure of thickeners as it is. Hurry and Cann applied AFM observation to examine unwashed grease surface and confirmed the dense, fibrous structure of thickener within the bulk [7].

This study investigated correlation between the structure formed by thickener fibers and the yield stress of the lithium soap greases with five different types of base oils. As the three-dimensional structure of thickeners is likely to be represented by size, shape, number and distribution of fibers, the thickener structures are evaluated quantitatively by analysis of fiber information obtained by two-dimensional AFM images for untreated grease surfaces. As the surface image of untreated greases obtained by AFM represents cross section of bulk greases, they are supposed to reflect three-dimensional structure.

\section{Experimental}

\subsection{Test greases}

The greases tested were composed of lithium 12-hydroxystearete thickener and five types of base oils of poly alpha olefin (PAO), carbonate ester (COE), polyol ester (POE) and two types of poly alkylene glycol (PAG) with the same range of viscosity and different relative dielectric constant. Their properties are shown in Table 1.

The greases were prepared by dissolving the thickener in the base oil at up to $503 \mathrm{~K}$, water-cooled by pouring it on a stainless tray, and then passing it through a three roll mill. Thickener concentration was 11 mass $\%$ and no additives were contained in the greases. Grease penetration was greatly changed with the base oils having different relative dielectric constant at the same thickener concentration. In addition to the five greases, greases with different thickener concentration were also prepared for three types of base oils of PAO, POE and PAG-3. The properties of these greases are shown in Table 2.

\subsection{Rheological measurement}

Rheological characteristics were evaluated by a rheometer with a parallel plate. Yield stress of the greases was measured at $303 \mathrm{~K}$ in oscillating tests. Shear stress was increased continuously from $10 \mathrm{~Pa}$ to $5000 \mathrm{~Pa}$ at 10 $\mathrm{Hz}$. Yield stress was defined as the shear stress value when loss tangent $G$ " $G$ ' became unity [5]. A viscous behavior of greases represented by loss modulus $G$ "' becomes dominant compared with an elastic behavior represented by storage modulus $G$ ' when loss tangent is greater than unity.

\subsection{AFM measurement}

Untreated surface of the greases was observed by AFM with non contact mode. A cantilever made of $\mathrm{Si}_{3} \mathrm{~N}_{4}$ was oscillated and controlled to put close to grease surface at a position where amplitude of oscillation decreases $40 \%$ of initial value in $10 \mu \mathrm{m}$ square with a scan speed of $0.5 \mathrm{~Hz}$ in ambient air. Immediately after grease was applied to flat glass in a thin layer, an AFM observation was conducted. Flat part of the grease surface was selected to obtain AFM image, because the grease surface had some undulations. Viscoelastic character of the surface reflects the response of the oscillation of a cantilever. An AFM image was obtained by analyzing this phase difference. Dark area on an AFM image represents elastic body of thickeners and light area the viscous region of base oil. Measurements were made

Table 1 Properties of greases

\begin{tabular}{|c|c|c|c|c|c|}
\hline Grease & PAO & COE & POE & PAG1 & PAG3 \\
\hline Base oil type & PAO & COE & POE & \multicolumn{2}{|c|}{ PAG } \\
\hline Base oil viscosity at 313K, $\mathrm{mm}^{2} / \mathrm{s}$ & 31 & 31.6 & 30.5 & 32.8 & 29.8 \\
\hline $\begin{array}{c}\text { Base oil relative dielectric constant } \\
\text { at 1MHz }\end{array}$ & 2.1 & 2.2 & 2.3 & 5.4 & 8.1 \\
\hline $\begin{array}{c}\text { Worked penetration at } 298 \mathrm{~K} \\
\text { (282 }\end{array}$ & 262 & 212 & 240 & 310 \\
\hline
\end{tabular}

Table 2 Properties of greases with different thickener concentration

\begin{tabular}{|c|ccc|cc|cc|}
\hline Grease & \multicolumn{3}{|c|}{ PAO } & \multicolumn{3}{c|}{ POE } & \multicolumn{2}{c|}{ PAG3 } \\
\hline Thickener concentration, $\%$ & 14 & 15 & 16 & 3 & 6 & 16 & 18 \\
\hline Worked penetration at 298K & 240 & 222 & 193 & 328 & 269 & 250 & 212 \\
\hline
\end{tabular}




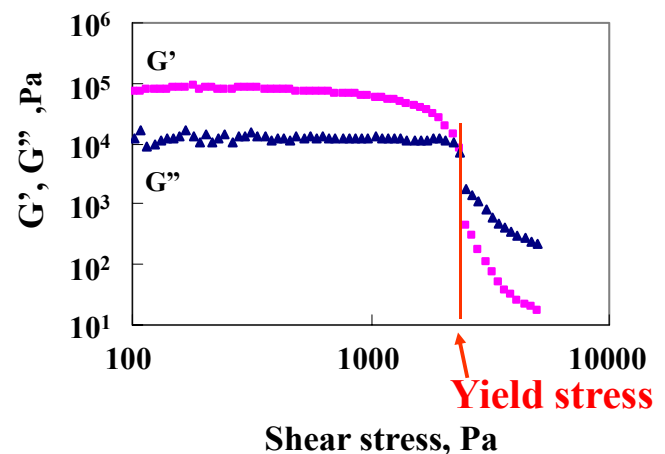

Fig. 1 A result of yield stress measurement of PAO

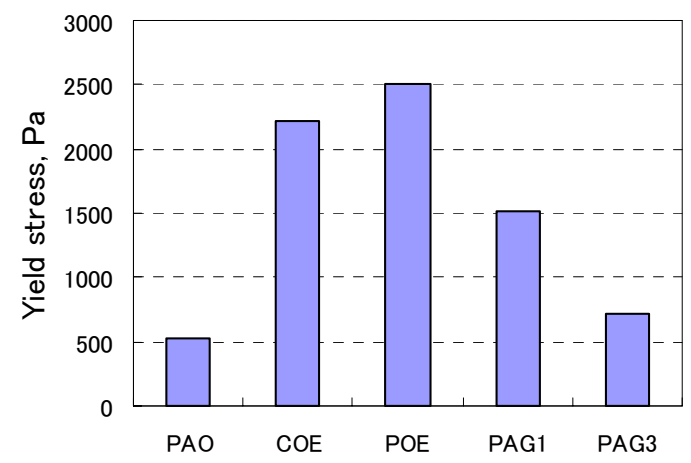

Fig. 2 Yield stress of tested greases

for two or three views for each grease, and they had the same tendency.

\section{Results and discussion}

\subsection{Yield stress of the greases}

A result of yield stress measurement of PAO is shown in Fig. 1. Variation of storage modulus $G^{\prime}$ against shear stress is greater than that of loss modulus $G$ ' and drastically decreased around the yield stress point. Figure 2 shows yield stress of the greases with the same thickener concentration but different type of base oil. The yield stress varied depending on the base oil types despite the thickener concentration was the same. The POE grease with small worked penetration indicated high yield stress and the PAG-3 grease with large worked penetration indicated low yield stress. It is likely that the difference of the rheological properties by the type of base oils is caused by the three-dimensional structure of greases and that the thickener structures are influenced by the base oil type.

\subsection{AFM observations}

Figure 3 shows AFM images of the five greases with the same thickener concentration of 11 mass $\%$ and different types of base oils. AFM images reveals that isolated thickeners are distributed in the greases and shape and distribution of the fibers are different for greases with different types of base oil. PAO grease has the shortest fiber length in the five greases. SEM images

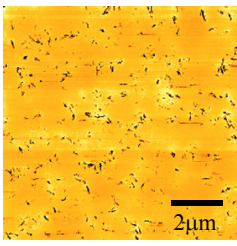

(a) PAO

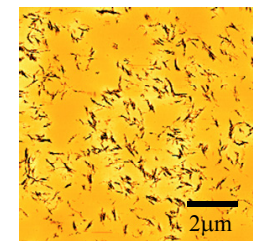

(b) $\mathrm{COE}$

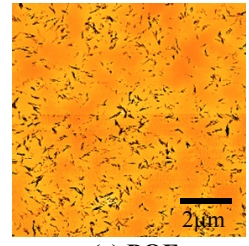

(c) POE

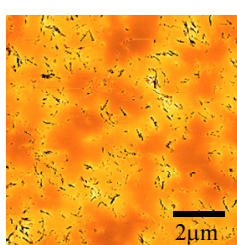

(d) PAG1

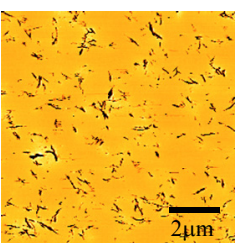

(e) PAG3
Fig. 3 AFM images of the greases with different type of base oil at same thickener concentration $(11 \%)$

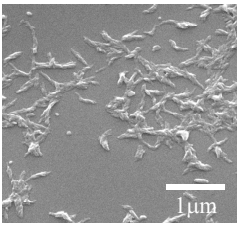

(a) PAO

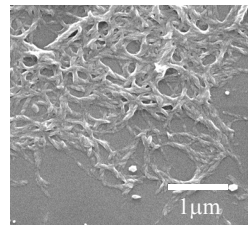

(b) $\mathrm{COE}$

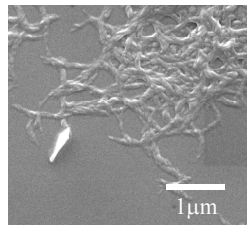

(c) POE

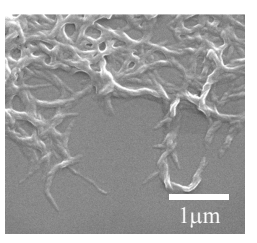

(d) PAG1

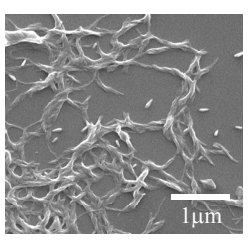

(e) PAG3
Fig. 4 SEM images of the greases with different type of base oil at same thickener concentration $(11 \%)$

of the same greases of Fig. 3 are shown in Fig. 4. The pretreatment of making dried thickeners after removing base oil by hexane was conducted for SEM analysis. As compared with the SEM images, each fiber shown in an AFM image looks isolated and their length is slightly short. It appears that the pretreatment for SEM observation has caused aggregation of the thickener fibers which are originally dispersed in the base oil.

AFM images of thickener distribution with different thickener concentration of PAO grease are shown in Fig. 5. The range of fiber length is almost the same for PAO greases with different thickener concentrations. Density of thickeners is greater with larger thickener concentration, and distribution of thickeners also seems to be different. Thickener fibers are distributed slightly inhomogeneously and, as indicated by an arrow $\mathrm{A}$ in the images, loosely closed area with no thickener can be observed. It appears that this area can be more clearly distinguished from denser part in the image for the greater thickener concentration in Fig. 5(b). This loosely 
closed area is formed by aggregation of isolated thickener fibers in the three-dimensional structure, and acts as a pocket to hold base oil in the grease. This closed area is also observed on the other greases with different base oils. These results clearly demonstrate that two-dimensional AFM images partially represent the three-dimensional structure formed by thickener fibers.

\subsection{Parameters for AFM images}

In order to discuss the thickener structures and its dependence on the rheological properties of the greases, a number of parameters listed in Table 3 are examined to represent the thickener structure in the AFM images. The thickener structures consist of the factors of both thickener fiber and aggregate of thickeners. These two factors are divided into five detailed parameters of size, shape, number, density and distribution. These parameters are evaluated by binarized data of the AFM images. Each AFM image is a square of $10 \mu \mathrm{m}$ by $10 \mu \mathrm{m}$ and expressed with $256 \times 256$ pixels. Each pixel is converted to grayscale with from 0 to 255 level of brightness before smooth continuous background is removed from fibers and other images based on the rolling ball algorithm described in reference [8]. Dark pixels for fibers in an image are converted to black, while other pixels to white according to a threshold value of the brightness determined by the isodata algorithm described in reference [9]. The threshold is defined as a value which is equal to the average of the average brightness (a) PAO, T.C. $11 \%$

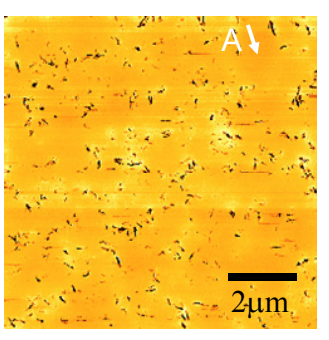

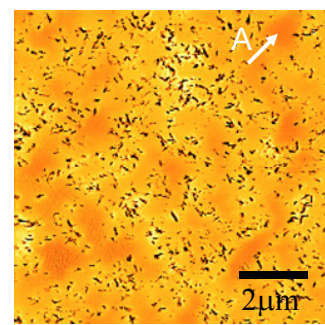

(b) PAO, T.C. 16\%
Fig. 5 AFM images of the greases with different thickener concentration of the PAO grease for those pixels brighter than the threshold and the average brightness for those pixels darker than the threshold. The fiber radius $r$ and circularity $\chi$ are defined as follows:

$$
\begin{aligned}
& r=\sqrt{\frac{S}{\pi}} \\
& \chi=4 \pi \times \frac{S}{L^{2}}
\end{aligned}
$$

where $S$ and $L$ are area and perimeter of each particle, respectively. Number of fibers $n$ is determined by counting black particles for fibers on a measured area and the density of thickeners $c$ is represented by the rate of sum of black pixels on a measured area of $256 \times 256$ pixels. Distance between particles $D i$ is determined by obtaining the average distance from the centroid of each particle to the nearest particle.

Relationship between yield stress and the five parameters are shown in Fig. 6. Coefficient of determination was calculated with power functions fitted by the least squares method. The parameters of the number of fibers, the rate of surface area of fibers and the interparticle distance had correlation with the yield stress to some degree, while the equivalent fiber radius and circularity shown in Fig. 6(a) and Fig. 6(b) showed no correlation. The value of the rate of surface area of fibers of the greases tended to be smaller than the prepared thickener concentrations. This is because thickeners of tested greases were aggregated to some extent in bulk grease as observed as asperities on the surface.

\subsection{The index of the degree of dispersion}

AFM images in Fig. 3 indicate that thickener structures are formed by aggregation of isolated fibers. Distribution of isolated fibers is supposed to become uniform when the structure are developed and thickens the grease completely. Therefore, the extent of development of the thickener structures can be evaluated by the difference of distribution of fibers from a complete dispersed state.

To evaluate the extent of development of the thickener structures, a new index of the degree of dispersion was defined as Eq. (3) [10]. Fiber shape was

\begin{tabular}{|c|c|c|c|c|}
\hline & Factor & & Index & Symbol \\
\hline \multirow{5}{*}{$\begin{array}{l}\text { Thickener } \\
\text { structure }\end{array}$} & \multirow{2}{*}{$\begin{array}{l}\text { Thickener } \\
\text { fiber }\end{array}$} & Size & Fiber radius & $r$ \\
\hline & & Shape & Circularity & $\chi$ \\
\hline & \multirow{3}{*}{$\begin{array}{l}\text { Aggregate of } \\
\text { thickeners }\end{array}$} & Number & Number of fibers & $n$ \\
\hline & & Density & $\begin{array}{l}\text { The proportion of } \\
\text { the area of fibers } \\
\text { in the AFM } \\
\text { projection image }\end{array}$ & $c$ \\
\hline & & Distribution & $\begin{array}{l}\text { Distance between } \\
\text { particles }\end{array}$ & $D i$ \\
\hline
\end{tabular}

Table 3 Factors of thickener structure 


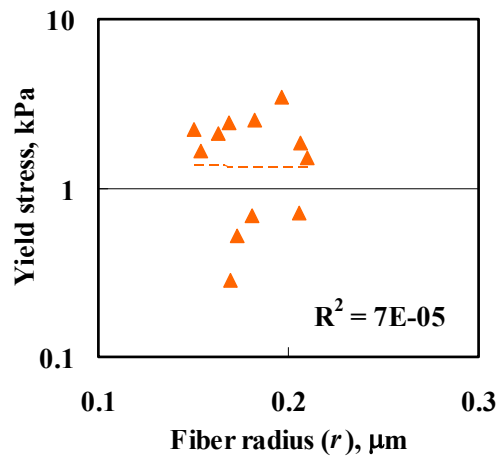

(a) Fiber size

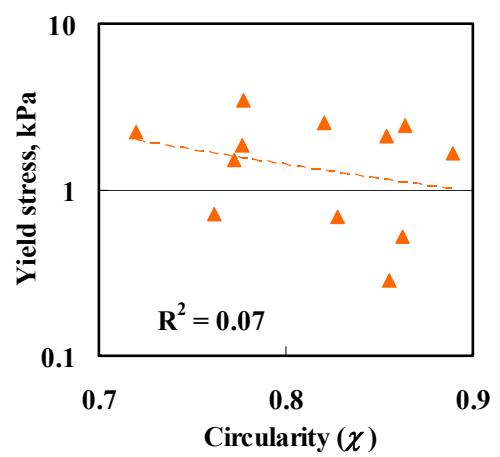

(b) Shape of fibers

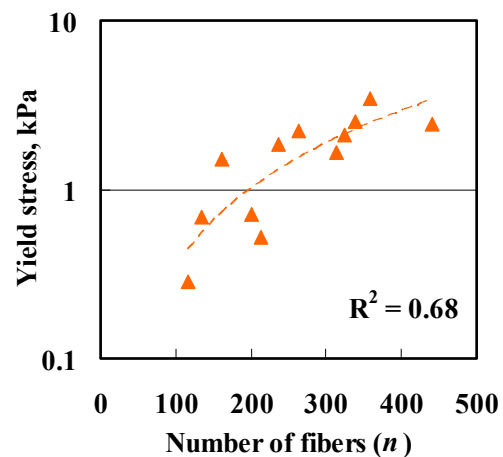

(c) Number of fibers

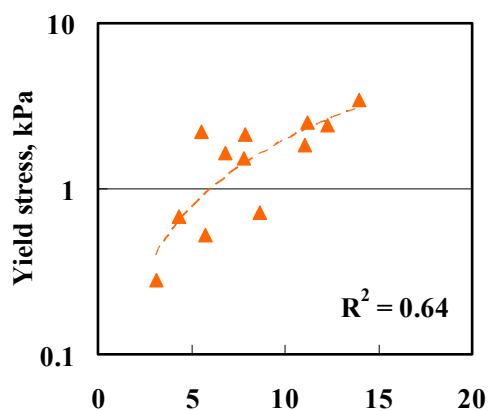

The proportion of the area of fibers in the AFM projection image $(c), \%$

(d) Density of fibers

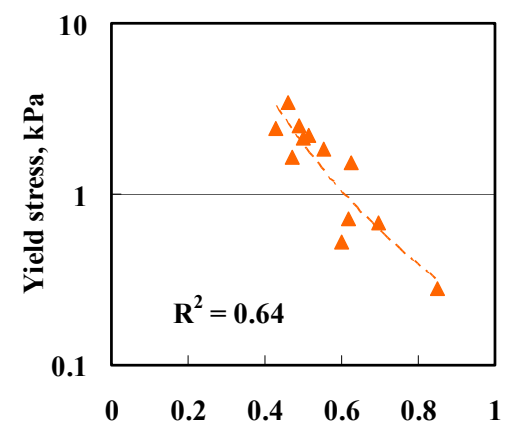

Distance between particles $(\mathrm{Di}), \mu \mathrm{m}$

(e) Distribution of fibers

Fig. 6 Relationship between yield stress and the factors of thickener structure

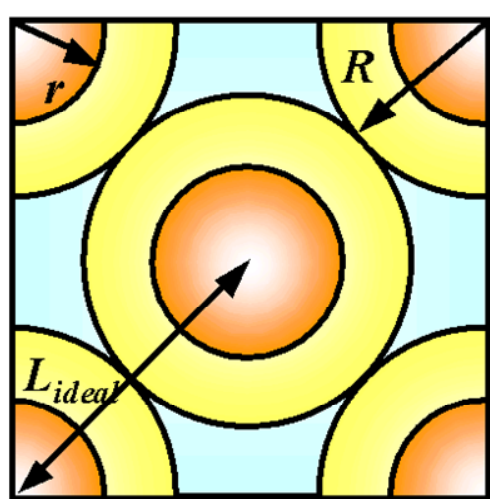

Fig. 7 Illustration to explain the degree of dispersion

assumed as circle to estimate a complete dispersed state of fibers. Figure 7 shows an illustration to explain the degree of dispersion.

The degree of dispersion $\Theta=\frac{\sum_{i=1}^{n} D i}{n \times D_{\text {ideal }}}$

where

$$
D_{\text {ideal }}=2 R=2 r \sqrt{\frac{\phi}{c}}
$$

$$
\begin{aligned}
& \phi \times \frac{A}{n}=\pi R^{2} \\
& n=\frac{A \times c}{\pi r^{2}}
\end{aligned}
$$

where $D i$ is distance between particles, $D_{\text {ideal }}$ is interparticle distance in close-packed structure, $n$ is number of fibers, $R$ is theoretical radius of hypothetical circle particle at close-packed structure, $\phi$ is filling rate at close-packed structure, $c$ is the proportion of the area of fibers in the AFM projection image and $A$ is measurement area. The degree of dispersion $\Theta$ represents the ratio of interparticle distance to the interparticle distance for a close-packed structure. This index includes information about the four thickener parameters of fiber size $r$, number of fibers $n$ and density of thickeners $c$ and distribution of thickeners on grease surface $D i$. Fiber size was represented by fiber radius, whereby the shape of thickeners was assumed as circle. The four parameters of thickener $r, n, c$ and $D i$ are obtained from AFM images. Filling factor $\phi$ for a close-packed structure was assumed as 0.68 of FCC. The degree of dispersion takes a value between 0 and 1 , and becomes 1 when thickeners distribute at closed-packed position.

Values of the degree of dispersion for PAO, COE, POE, PAG1 and PAG3 grease were 0.59, 0.61, 0.67, 0.59 


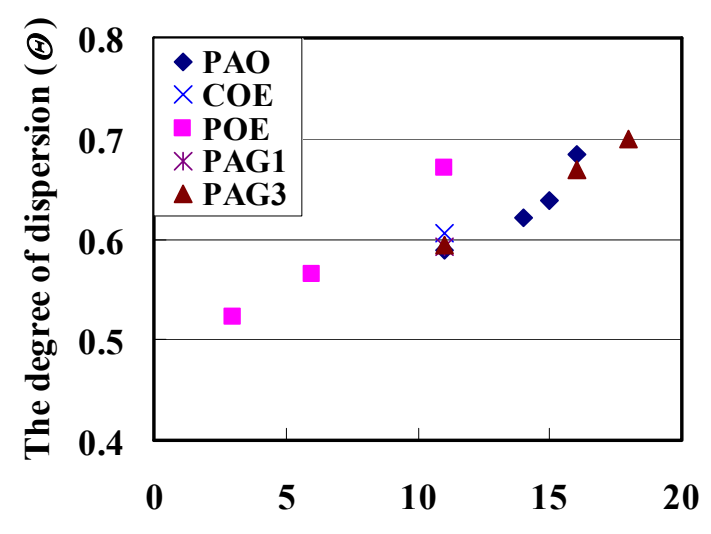

Thickener concentration, $\operatorname{mass} \%$

Fig. 8 Relationship between the degree of dispersion and thickener concentration

and 0.59 , respectively. This suggests thickeners of the POE grease, which has the largest yield stress, form the most uniform thickener structures in the five greases, whereas the degree of dispersions of PAO, COE, PAG1 and PAG3 are almost the same.

\subsection{Relationship between the degree of dispersion and yield stress}

Figure 8 shows relationship between the degree of dispersion and thickener concentration for the five types of greases. The degree of dispersion ranged from 0.52 to 0.70 . The PAO, POE and PAG3 greases show higher degree of dispersion for greater thickener concentration. This quantitatively indicates the homogenization of thickener distribution with the increase in thickener concentration.

Figure 9 shows the relationship between the degree of dispersion and the yield stress of the tested twelve greases. The degree of dispersion has good correlation with the yield stress. The yield stress become larger with the increase in the degree of dispersion and its correlation coefficient was 0.80 . Thus this index represents a quantitative measure of the thickener structure which determines the yield stress. The degree of dispersion is employed to express the thickener structures in the presence of the base oil, and it contains information about size, number, density and distribution of the thickeners. Relationships between thickener structures and physical properties have long been argued but only qualitatively, and this new index is the first one that allows us to make quantitative examination. As this study aims to show the capability of the new parameter, the influence of the base oil type on the rheological property is not shown in detail. More detailed analysis of the interaction between thickeners and base oils will be addressed in the future work.

\section{Conclusions}

The relationship between the structure formed by thickeners and the yield stress of lithium soap greases

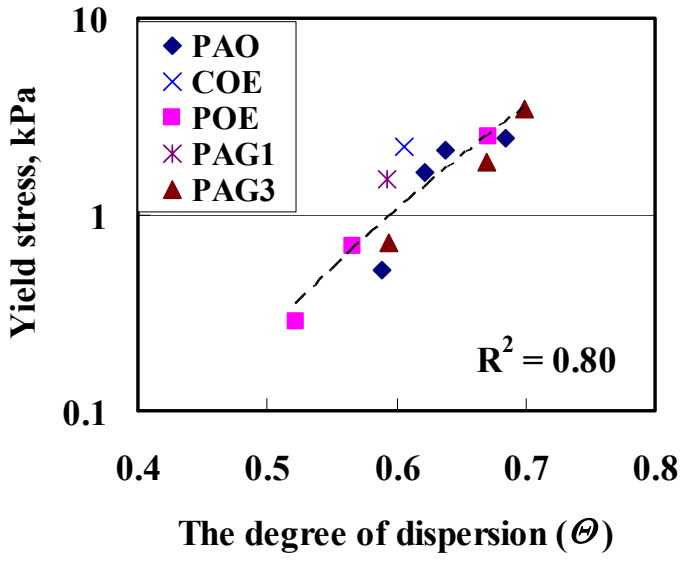

Fig. 9 Relationship between the degree of dispersion and yield stress

with five types of base oils were studied in this paper. The thickener structures are evaluated quantitatively by analyzing the images obtained with an atomic force microscope. The results are summarized as follows.

(1) AFM images for lithium soap greases with the base oils of PAO, POE and PAG showed the development of the structure formed by thickeners with greater thickener concentration.

(2) The degree of dispersion obtained from an AFM image is proposed to describe the structure formed by thickeners quantitatively.

(3) Greases with more uniform distribution of the thickeners, which is indicated by a larger value of the degree of dispersion, have higher yield stress.

\section{References}

[1] Yokouchi, A., Hokao, M. and Sugimura, J., "Effects of Soap Fiber Structure on Boundary Lubrication of Lithium Soap Greases," Tribology Online, 6, 4, 2011, 219-225.

[2] Oikawa, E., Inami, N., Hokao, M., Yokouchi, A. and Sugimura, J., "Bearing Torque Characteristics of Lithium Soap Greases with Some Synthetic Base Oils," J. of Engineering Tribology, 226, 6, 2012, 575-583.

[3] Cann, P. M. and Spikes, H.A., "Fourier-Transform Infrared Study of the behavior of grease in Lubricated Contacts," Lubrication Engineering, 48, 4, 1992, 335-343.

[4] Meng, Y. and Zheng, J., “A Rheological Model for Lithium Lubricating Grease," Tribology International, 31, 10, 1998, 619-625.

[5] Couronné, I., Blettner, G. and Vergne, P., "Rheological Behavior of Grease: Part I - Effects of Composition and Structure," Tribology Transactions, 43, 4, 2000, 619-626.

[6] Delgado, M. A., Valencia, C., Sánchez, M. C., Franco, J. M. and Gallegos, C., "Influence of Soap Concentration and Oil Viscosity on the Rheology 
and Microstructure of Lubricating Greases," Industrial \& Engineering Chemistry Research, 45, 6, 2006, 1902-1910

[7] Hurly, S. and Cann, P. M., "Examination of Grease Structure by SEM and AFM Techniques," NLGI Spokesman, 65, 5, 2001, 17-26.

[8] Stanley, S. R., "Biomedical Image Processing," IEEE Computer, 16, 1, 1983, 22-34.
[9] Ridler T. W. and Calvard S., "Picture Thresholding Using an Iterative Selection Method," IEEE Trans. System, Man and Cybernetics, SMC-8, 1978, 630-632.

[10] Sasakura, H. and Yamaguchi, U., "3D Analysis of Polymer Nanocomposite by Using TEM-Computerized Tomography," Function \& Materials, 25, 8, 2005, 5-11 (in Japanese). 